\title{
Long Non-Coding RNA Deregulation in Tongue Squamous Cell Carcinoma
}

\author{
Wei Gao, Jimmy Yu-Wai Chan, and Thian-Sze Wong \\ Department of Surgery, LKS Faculty of Medicine, The University of Hong Kong, Queen Mary Hospital, \\ 102 Pokfulam Road, Kowloon, Hong Kong
}

Correspondence should be addressed to Thian-Sze Wong; thiansze@graduate.hku.hk

Received 24 April 2014; Accepted 29 May 2014; Published 22 June 2014

Academic Editor: Changyang Gong

Copyright (C) 2014 Wei Gao et al. This is an open access article distributed under the Creative Commons Attribution License, which permits unrestricted use, distribution, and reproduction in any medium, provided the original work is properly cited.

Background. The deregulated tumorigenic long non-coding RNA (lncRNA) has been reported in several malignancies. However, there is still no comprehensive study on tongue squamous cell carcinoma (SCC). Methods. Functional reannotation for the human lncRNA was carried out by ncFANs. Real-time quantitative PCR was used to validate the identified lncRNAs. Results. Using the functional annotation algorithm from ncFANs, 8 differentially expressed lncRNAs were identified. Lnc-PPP2R4-5, lnc-SPRR2D-1, lnc-MAN1A2-1, lnc-FAM46A-1, lnc-MBL2-4:1, and lnc-MBL2-4:3 were upregulated in the microdissected tongue SCC tissues. In comparison, lnc-AL355149.1-1 and lnc-STXBP5-1 showed significant downregulation. High level of lnc-MBL2-4:3 was significantly associated with the node positive tongue SCC patients. Further, patients with advanced T-stage demonstrated a further reduction of lnc-AL355149.1-1 in the tumor tissues. Treatment of tongue SCC cells with 5-fluorouracil and paclitaxel can reserve the expression patterns observed in the tongue SCC tissues. Further, changes of lnc-MBL2-4:3 and lnc-AL355149.1-1 expression levels were noticed in the cisplatin-resistant tongue SCC cells. Conclusions. Our results demonstrated that functional reannotation allows us to identify novel lncRNAs using the existing gene expression array dataset. The association of lncRNA with the T-stage and nodal status of tongue SCC patients suggested that lncRNA deregulation was involved in the pathogenesis of tongue SCC.

\section{Introduction}

Tongue squamous cell carcinoma (SCC) is a common epithelial cancer identified in the oral cavity. In USA, tongue is the most common site of oropharyngeal cancer (in comparison with mouth, pharynx, and others in oral cavity) and the major histological form is squamous cell carcinoma [1]. Major causative factors included tobacco consumption and alcohol abuse. Viral infection including human papilloma virus and Epstein-Barr virus infection might also play a part in the development of tongue SCC [2]. Tongue SCC is an aggressive tumor with rapid growth rate and high chance of regional and distant metastasis. Tumor dimension and the existence of extracapsular spread (ECS) are predictors of survival [3]. In addition, regional spreading to the cervical lymph node and distant metastasis of tongue SCC are indicators of poor prognosis $[4,5]$.

Different from the mRNA transcript, the codon on the long non-coding RNA (lncRNA) do not code for any peptides or proteins. lncRNA refers to RNA molecules with size over 200 b.p. long and without protein coding functions. To date, the precise control mechanisms of lncRNA are not completely understood. LncRNA could be transcribed by RNA polymerases I, II, and III [6]. LncRNA functions as epigenetic regulators in the somatic cells and is directly involved in cell cycle regulation and cell differentiation [7]. Although the precise regulatory mechanism of lncRNA is not fully understood, evidence suggested that expression of certain IncRNA is being modulated by external stimulus, such as cellular irradiation and chemotherapeutic agents treatment $[7,8]$. Further, lncRNA is alleged to be involved in the development of resistant phenotype and deters the efficacy of cancer treatment [9].

To the best of our knowledge, comprehensive lncRNA study aiming at identifying novel lncRNA signature has not yet been carried out in the tongue squamous cell carcinoma. Hence, in the present study, we aimed at identifying the candidate lncRNA associated with tongue SCC using the 
TABle 1: Primer and probe sequences for qPCR.

\begin{tabular}{|c|c|c|c|c|}
\hline Vega ID & LNCipedia ID & Forward primer $\left(5^{\prime}-3^{\prime}\right)$ & Reverse primer $\left(5^{\prime}-3^{\prime}\right)$ & UPL number* \\
\hline OTTHUMG00000020778 & lnc-PPP2R4-5 & tggattttcatgcctgctg & ggctgcattaccagaaaggt & 3 \\
\hline OTTHUMG00000012449 & lnc-SPRR2D-1 & gcctctcctgcaagtgtga & tcctcatttatgacattttcagtctc & 5 \\
\hline OTTHUMG00000012147 & lnc-MAN1A2-1 & gagaccgaggaatcttgctg & ctcagtgggctcagtaatgct & 21 \\
\hline OTTHUMG00000015099 & lnc-FAM46A-1 & aggggtctcttgtccttggt & atcctcttattggcacactgc & 26 \\
\hline OTTHUMT00000048111 & lnc-MBL2-4:1 & gcagccctggagagtttatct & cagcataatatggatgtttgaagg & 67 \\
\hline OTTHUMT00000048112 & lnc-MBL2-4:3 & gagccagcaaaggagactga & cccagaaggggctcttactc & 36 \\
\hline OTTHUMG00000002490 & lnc-AL355149.1-1 & gaaaactaggcgtctgggaac & caaacaatgggagcaagtcc & 25 \\
\hline OTTHUMG00000015764 & lnc-STXBP5-1 & gctatgggaattatttttcctgtg & ggtaagccagttttcccttttt & 16 \\
\hline
\end{tabular}

${ }^{*}$ Probe number in the universal probe library.

microarray dataset available in the public microarray data repository. Real-time quantitative PCR was then used to confirm the expression and validate the results in the primary tongue SCC tissues and the paired normal epithelia. We also correlated the expression patterns with the clinical characteristics of tongue SCC patients in order to reveal any potential clinical use of the lncRNA.

\section{Materials and Methods}

2.1. Microarray and Functional Reannotation. Microarray data set GSE9844 containing 38 microarray data (http://www. ncbi.nlm.nih.gov/geo/query/acc.cgi?acc=GSE9844/) was obtained from Gene Expression Omnibus (GEO). The dataset contains 26 microdissected tongue squamous cell carcinoma tissues and 12 control tissues examined with Human Genome U133 (HG-U133) Plus 2.0 array (Affymetrix).

2.2. Functional Reannotation. The probes on the HG-U133 Plus 2.0 array were reannotated for human lncRNA using non-coding RNA function annotation server (ncFANs) as described [10]. Differential expressed lncRNAs were selected using Student's $t$-test. A $P$ value below 0.05 was considered as differentially expressed lncRNAs. The $P$ value of differential expression was adjusted with the Benjamini and Hochberg correction for multiple comparisons.

2.3. Patient Samples. Tongue SCC patients were recruited at Department of Surgery, The University of Hong Kong, Queen Mary Hospital, Hong Kong. Written consent of tissue donation for research purposes was obtained from patients before tissue collection. The protocol was approved by the IRB of the hospital (IRB reference number UW 12-123). Paired tumor and normal tissues $(n=32)$ were collected. Histological confirmation of the tissues was performed by hospital's pathologists.

2.4. Real-Time Quantitative PCR. Total RNA was extracted and purified from the frozen tissues using TRIzol reagent (Invitrogen). The quality and quantity of the isolated RNA were examined with Nanodrop. Reverse transcription was carried out using cDNA conversion kit (Invitrogen). Primers for lncRNA detection and quantification were designed at Universal ProbeLibrary Assay Design Center (http://www.roche-applied-science.com/). The LNA-labeled probe was obtained from the Universal Probe Library (Roche Applied Science). The lncRNA transcript levels were measured by LightCycler 480 (Roche Applied Science) and normalized with the GAPDH levels using $2^{-\triangle \mathrm{Ct}}$ algorithm. Primer and probe sequences were listed in Table 1.

2.5. Cell Culture and Drug Treatment. Tongue SCC cell line HN21B was cultured in RPMI 1640 medium supplied with $10 \%$ fetal bovine serum (Gibco), $200 \mathrm{Unit} / \mathrm{mL}$ penicillin $\mathrm{G}$ sodium (Gibco), $200 \mu \mathrm{g} / \mathrm{mL}$ streptomycin Sulfate (Gibco), and $0.5 \mu \mathrm{g} / \mathrm{mL}$ amphotericin B (Gibco). The cell line was incubated in humidified incubator with $5 \% \mathrm{CO}_{2}$ at $37^{\circ} \mathrm{C}$. 5Fluorouracil (5-Fu) and paclitaxel were obtained from sigma. Stock solutions were prepared by dissolving the drugs in dimethyl sulfoxide (DMSO) at the concentration of $100 \mathrm{mM}$ and $1.2 \mathrm{mM}$ respectively. Cisplatin (Sigma) was dissolved in distilled water at a concentration of $3.3 \mathrm{mM}$. The stock solutions were freshly diluted to appropriate concentrations in RPMI 1640 medium before treatment. HN21B cells were treated by $0-10 \mu \mathrm{M} 5-\mathrm{Fu}, 0-8 \mathrm{nM}$ paclitaxel, or $0-64 \mu \mathrm{M}$ cisplatin for 72 hours followed by in vitro toxicity test. The toxicity test was performed using the Toxicology Assay Kit Sulforhodamine B (SRB) assay (Sigma-Aldrich) according to manufacturer's manual. The inhibitory concentration (IC) was calculated from the dose-response curve of HN21B upon drug treatment.

2.6. Development of Cisplatin-Resistant HN21B Cell Line. The cisplatin-resistant HN21B cell line was developed by chronic cisplatin treatment. HN21B cells were exposed to cisplatin for 3 days, followed by growth recovery in drugfree medium. The concentration of cisplatin was increased in the subsequent cycle and the procedure was repeated until resistance was achieved in HN21B cells.

2.7. Immunocytochemistry. $\mathrm{HN} 21 \mathrm{~B}$ cells were seeded on glass slides and fixed with $4 \%$ paraformaldehyde. The nucleus was stained by blue-fluorescent $4^{\prime}, 6$-diamidino-2-phenylindole (DAPI) (Invitrogen); F-actin was labeled in red with Alexa Fluor 635 phalloidin (Invitrogen).

2.8. Statistical Analysis. Statistical analysis was performed using SPSS V16.0 (SPSS, Chicago, IL). The statistical difference between tongue SCC and paired normal epithelia 

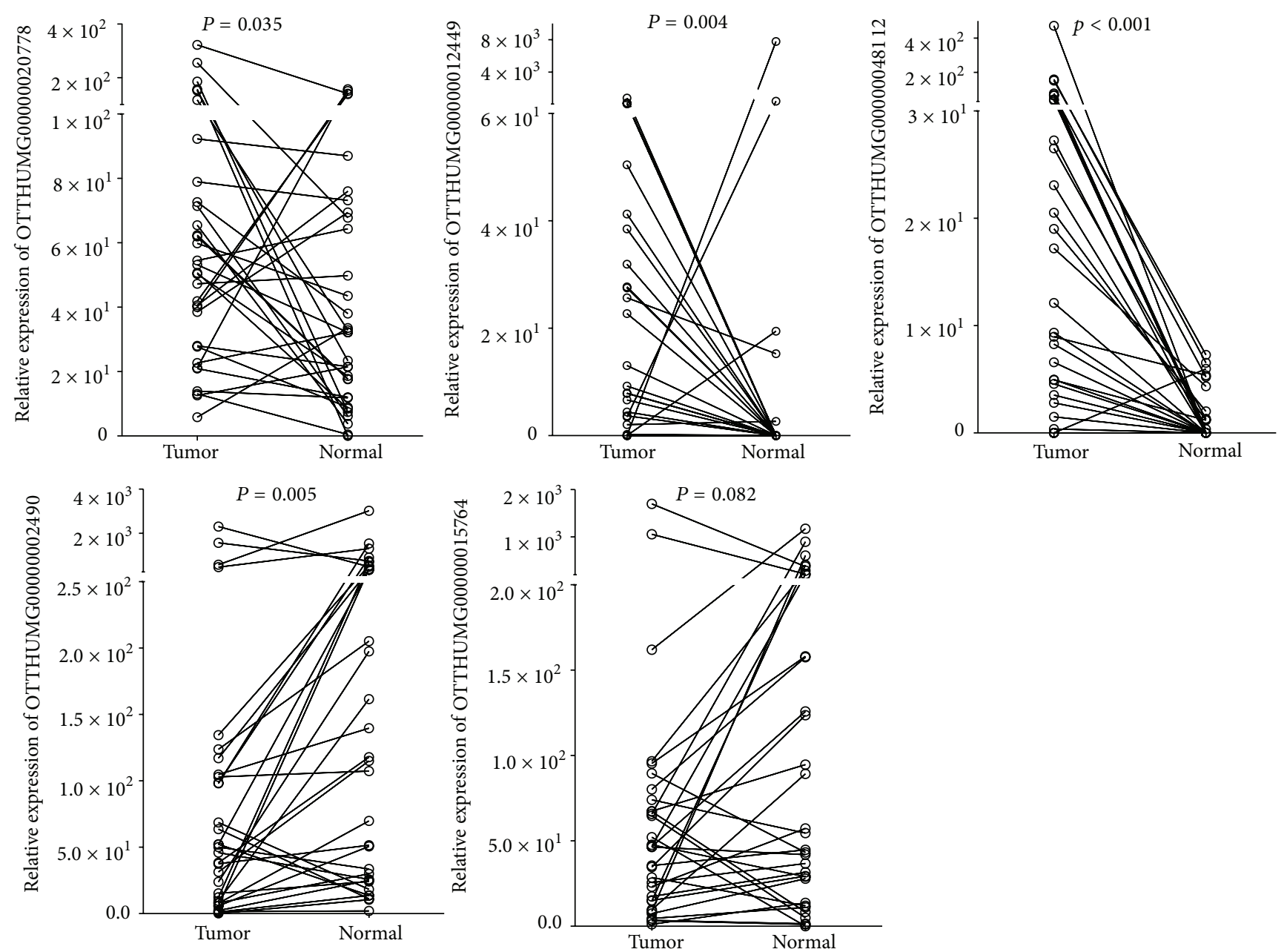

FIGURE 1: Relative expression levels of lncRNAs in paired tumor and normal tissue samples from patients with tongue SCC. The relative expression levels were normalized to GAPDH by qPCR analysis and the data are displayed as $2^{-\Delta \mathrm{Ct}}$. The difference between tumor and normal was calculated using the Wilcoxon signed-rank test.

was examined using Wilcoxon signed-rank test. All the tests were two-sided. $P$ value $<0.05$ was considered as statistically significant.

\section{Results}

3.1. Differential Expressed lncRNA in Tongue Squamous Cell Carcinoma. Using the functional annotation algorithm from ncFANs, 8 differentially expressed lncRNAs were identified (Table 2). Of the 8 lncRNA, lnc-PPP2R4-5, lnc-SPRR2D1, lnc-MAN1A2-1, lnc-FAM46A-1, lnc-MBL2-4:1, and lncMBL2-4:3 were upregulated in the microdissected tongue SCC tissues. In comparison, lnc-AL355149.1-1 and lncSTXBP5-1 showed significant downregulation in the tongue SCC.

3.2. Validation of $\ln c R N A$ by Real-Time Quantitative PCR. Of the 8 lncRNAs, lnc-MAN1A2-1 (OTTHUMG00000012147) was not detectable in all tissue samples. Lnc-MBL2-4:1 (OTTHUMT00000048111) was detectable in 3\% (1/32) tumor tissue samples and was not detectable in all the normal tissue samples. Lnc-FAM46A-1 (OTTHUMG00000015099) was detectable in $22 \%(7 / 32)$ of tumor tissue samples and $13 \%$ of (4/32) normal tissue samples.

For the remaining $5 \operatorname{lncRNAs}, 3$ were found to be significantly upregulated in the primary tongue SCC tissues. Overexpression of lnc-SPRR2D-1 (OTTHUMG00000012449) and lnc-PPP2R4-5 (OTTHUMG00000020778) was found in the tongue SCC tissue $(P=0.004 \& 0.035$ resp., Wilcoxon signed-rank test). Overexpression of the lnc-MBL2-4:3 (OTTHUMT00000048112) in the tongue SCC was highly significant in comparison with the paired normal epithelia $(P<0.001$, Wilcoxon Signed Ranked test). Lnc-AL355149.11 (OTTHUMG00000002490) was the only lncRNA found to be downregulated in the tongue SCC tissues $(P=$ 0.005 , Wilcoxon signed-rank test). The fold-change patterns (upregulated/downregulated) in the tongue tissues matched the fold-change patterns in the microarray dataset (Figure 1).

3.3. Correlations with Clinicopathological Parameters of Tongue SCC Patients. The expression levels of the 4 differentially expressed lncRNAs were correlated with the clinical 


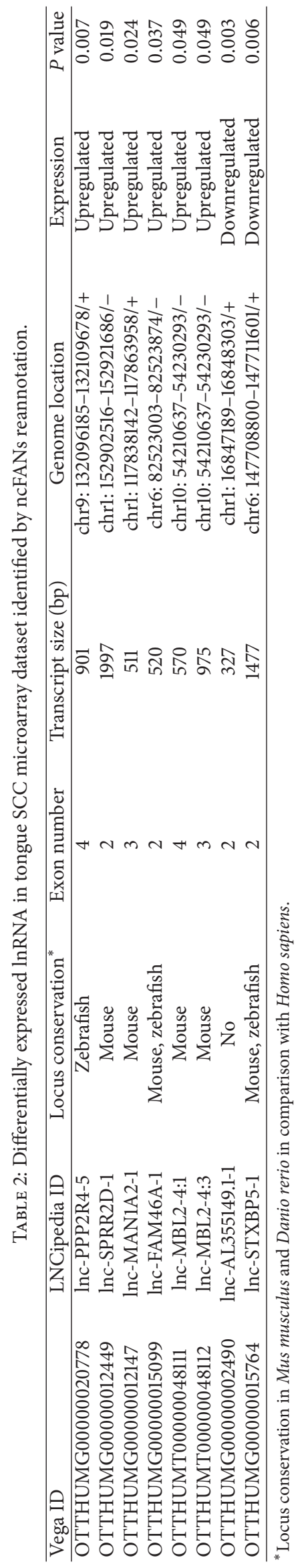


TABLE 3: Association of lncRNA expression levels with the clinicopathological variables in patients with tongue SCC.

\begin{tabular}{|c|c|c|c|c|c|}
\hline & & OTTHUMG00000020778 & OTTHUMG00000012449 & OTTHUMT00000048112 & OTTHUMG00000002490 \\
\hline \multicolumn{6}{|l|}{ Gender } \\
\hline Male & 18 & 0.866 & 0.206 & 0.338 & 0.099 \\
\hline Female & 14 & & & & \\
\hline \multicolumn{6}{|l|}{ Age } \\
\hline$<55$ & 16 & 0.270 & 0.254 & 0.809 & 0.669 \\
\hline$>55$ & 16 & & & & \\
\hline \multicolumn{6}{|l|}{ T-stage } \\
\hline T1-2 & 15 & 0.246 & 0.153 & 0.766 & $<0.001^{*}$ \\
\hline T3-4 & 17 & & & & \\
\hline \multicolumn{6}{|l|}{ Nodal stage } \\
\hline Negative & 18 & 0.925 & 0.065 & $0.002^{*}$ & 0.145 \\
\hline Positive & 14 & & & & \\
\hline \multicolumn{6}{|l|}{ Smoker } \\
\hline Yes & 17 & 0.766 & 0.628 & 0.295 & 0.526 \\
\hline No & 15 & & & & \\
\hline \multicolumn{6}{|l|}{ Drinker } \\
\hline Yes & 12 & 0.552 & 0.477 & 0.387 & 0.387 \\
\hline No & 20 & & & & \\
\hline
\end{tabular}

${ }^{*} P$ value below 0.05 was considered as statistical significance.

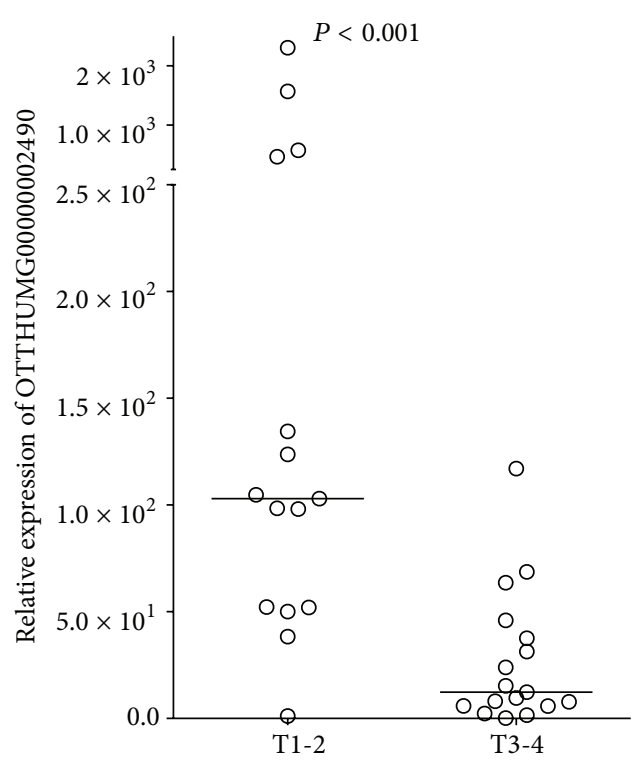

(a)

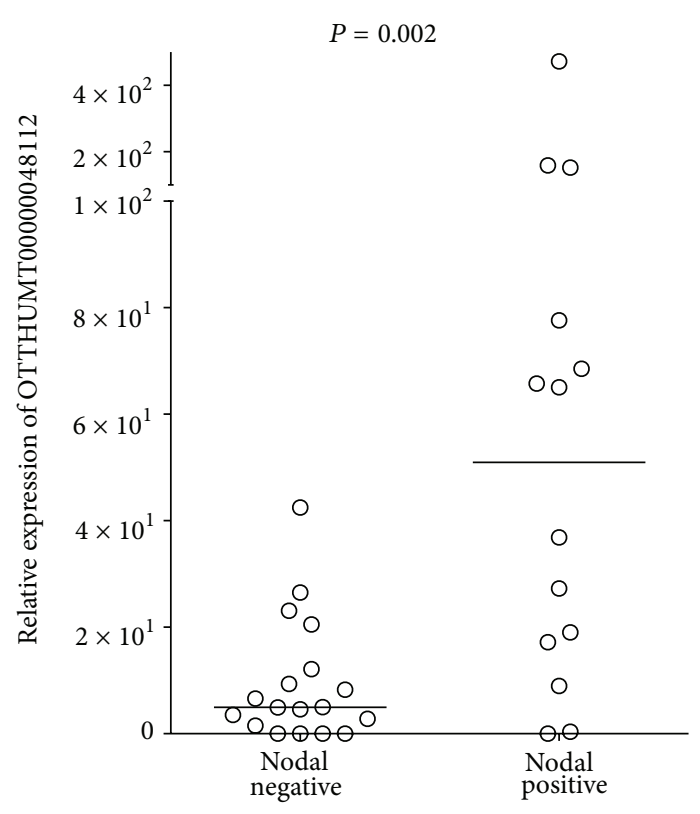

(b)

FIGURE 2: Relative expression levels of lncRNAs in tongue SCC patients with different T-stage (a) and nodal status (b). The relative expression levels were normalized to GAPDH by qPCR analysis. The difference was calculated using the Mann-Whitney $U$ test.

parameters of the patients (Table 3). High level of lnc-MBL24:3 (OTTHUMT00000048112) was significantly associated with the node positive tongue cancer patients compared to the node-negative patients $(P=0.002$, Mann-Whitney $U$ test). Further, lnc-AL355149.1-1 (OTTHUMG00000002490), the downregulated lncRNA in tongue SCC tissues, displayed substantial downregulation when the cancer progressed from early (T1-2) to advanced stages (T3-4). Figure 2 showed the expression difference of lnc-MBL2-4:3 and lnc-AL355149.11 in the tongue SCC tissues according to the T-stage and regional nodal status.

3.4. 5-Fu and Paclitaxel Treatment. To explore the potential functional role of the 2 identified lncRNAs with close 


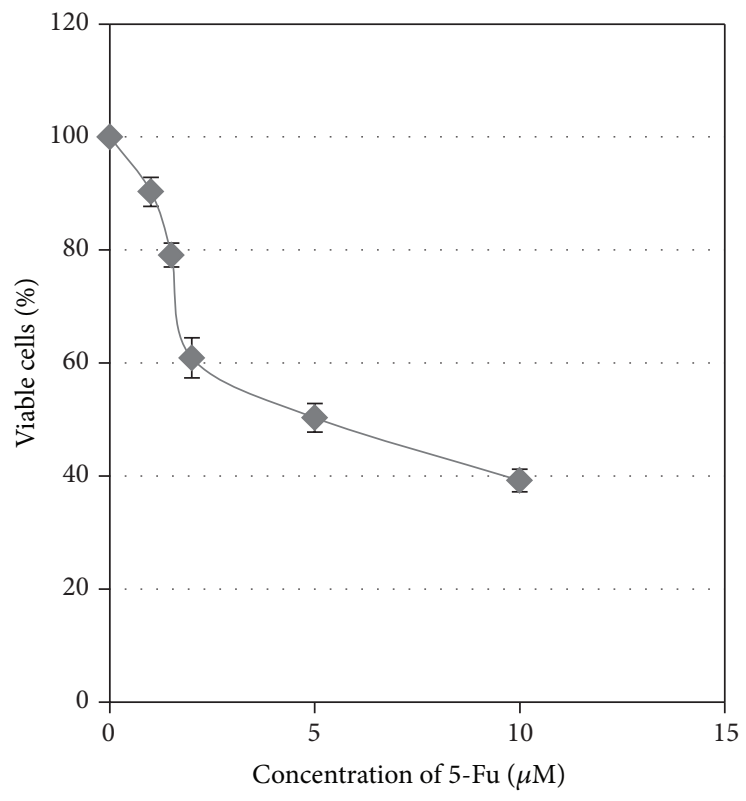

(a)

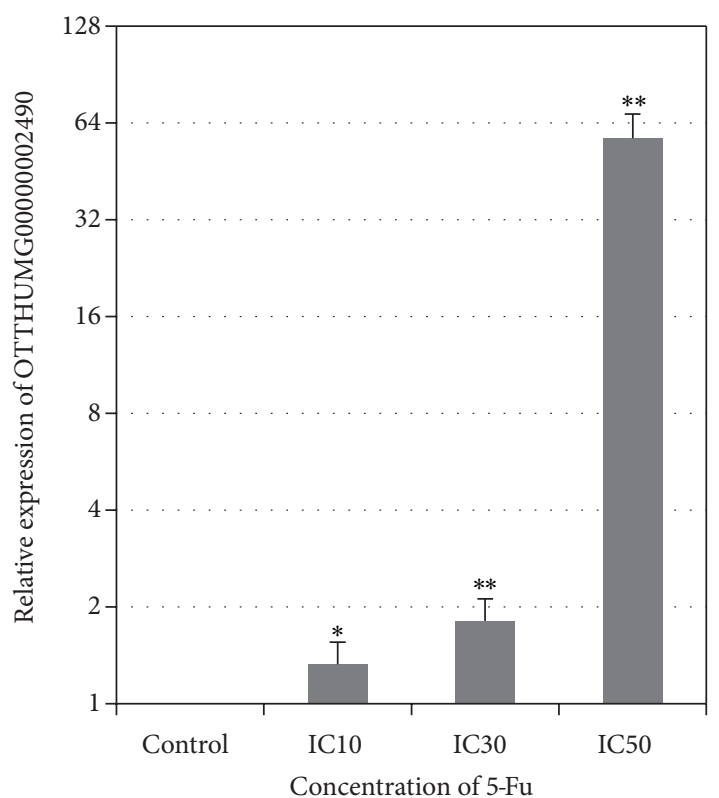

(b)

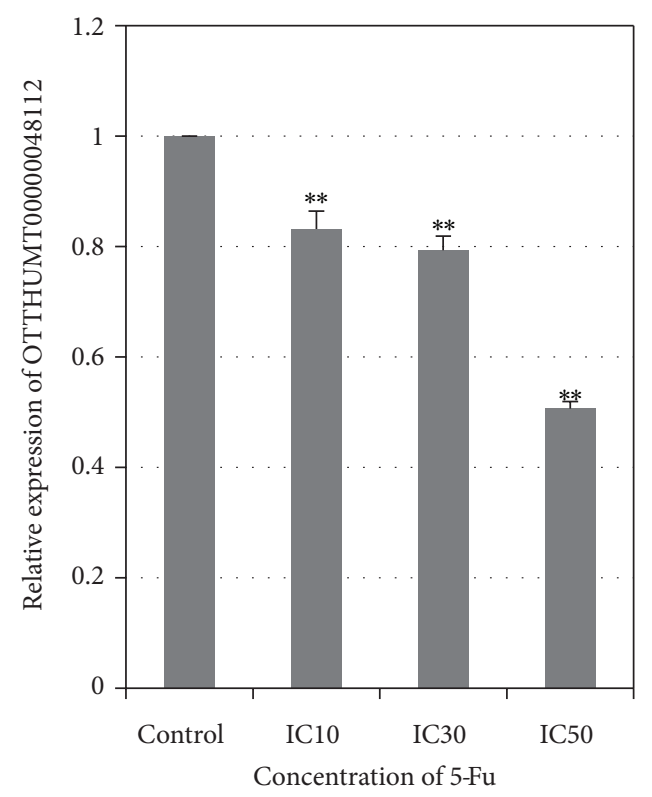

(c)

FIGURE 3: Effects of 5-Fu treatment on the expression of lncRNA in HN21B cells. (a) Dose-response curve of HN21B upon 5-Fu treatment. (b) and (c) Effect of 5-Fu treatment on the expression of OTTHUMG00000002490 and OTTHUMT00000048112, respectively. Bar, SD; $n=3$; ${ }^{*} P<0.05$ and ${ }^{* *} P<0.01$ by $t$-test.

correlation with the clinical features of the tongue SCC patients, we treated the cancer cell HN21B with 5-Fu and paclitaxel and examined the subsequent changes of lnc-MBL2-4:3 (OTTHUMT00000048112) and lncAL355149.1-1 (OTTHUMG00000002490) in response to the drug treatment. We use toxicity test to determine the inhibitory concentrations of 5-Fu and paclitaxel on tongue SCC cell line HN21B. For $5-\mathrm{Fu}$, the $\mathrm{IC}_{10}, \mathrm{IC}_{30}$, and $\mathrm{IC}_{50}$ were $0.6 \mu \mathrm{M}, 2.2 \mu \mathrm{M}$, and $4.3 \mu \mathrm{M}$, respectively (Figure 3(a)).
5-Fu treatment enhanced the expression of lnc-AL355149.11 (OTTHUMG00000002490), while it suppressed the expression of lnc-MBL2-4:3 (OTTHUMT00000048112) in a dose-dependent manner (Figure 3); for paclitaxel, the $\mathrm{IC}_{10}, \mathrm{IC}_{30}$, and $\mathrm{IC}_{50}$ on $\mathrm{HN} 21 \mathrm{Bc}$ were $0.1 \mathrm{nM}$, $1.0 \mathrm{nM}$, and $2.2 \mathrm{nM}$, respectively (Figure $4(\mathrm{a})$ ). Similar to the 5-Fu treatment, the expression of lnc-AL355149.1-1 (OTTHUMG00000002490) was activated upon paclitaxel treatment (Figure 4(b)). In addition, the expression of 


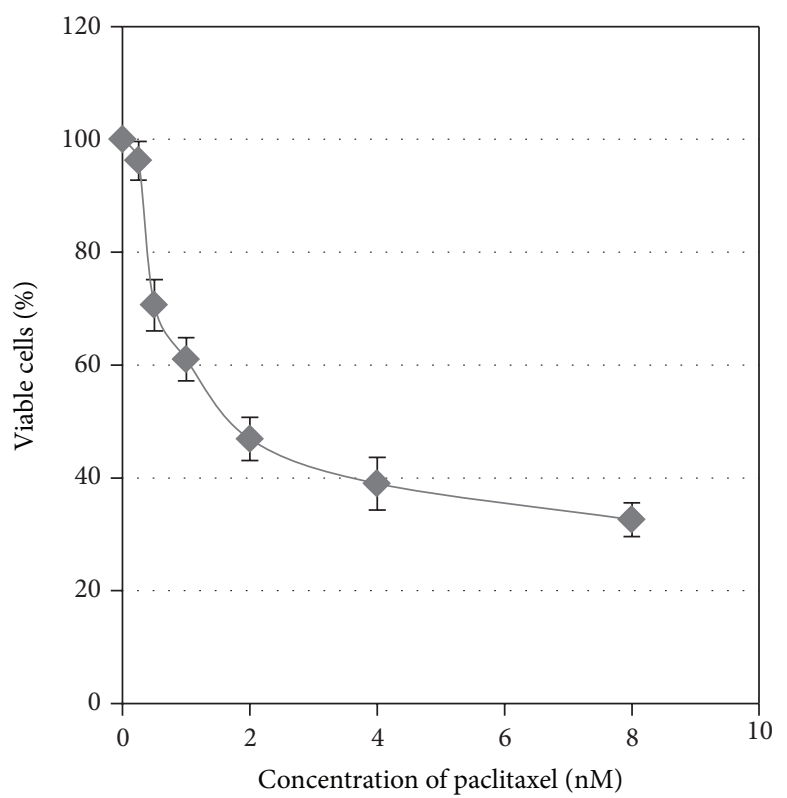

(a)

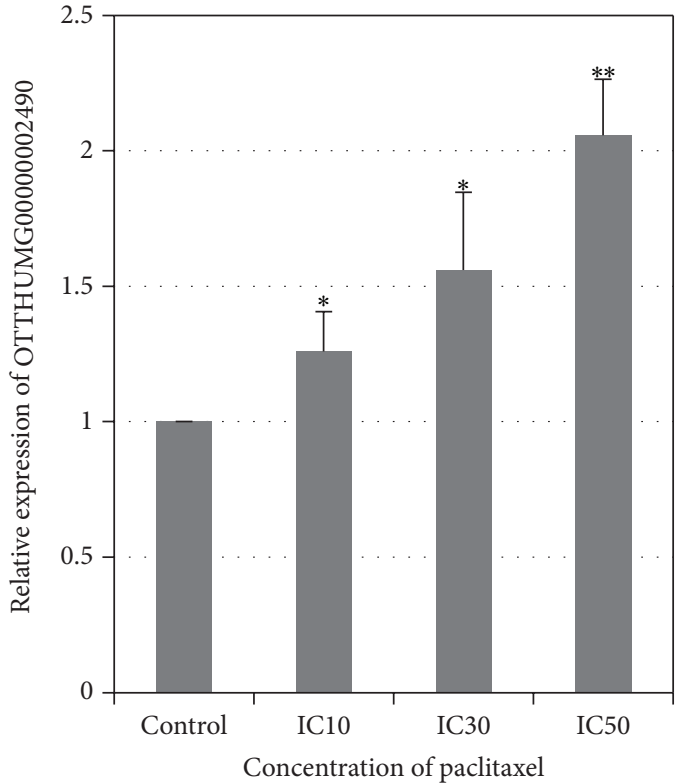

(b)

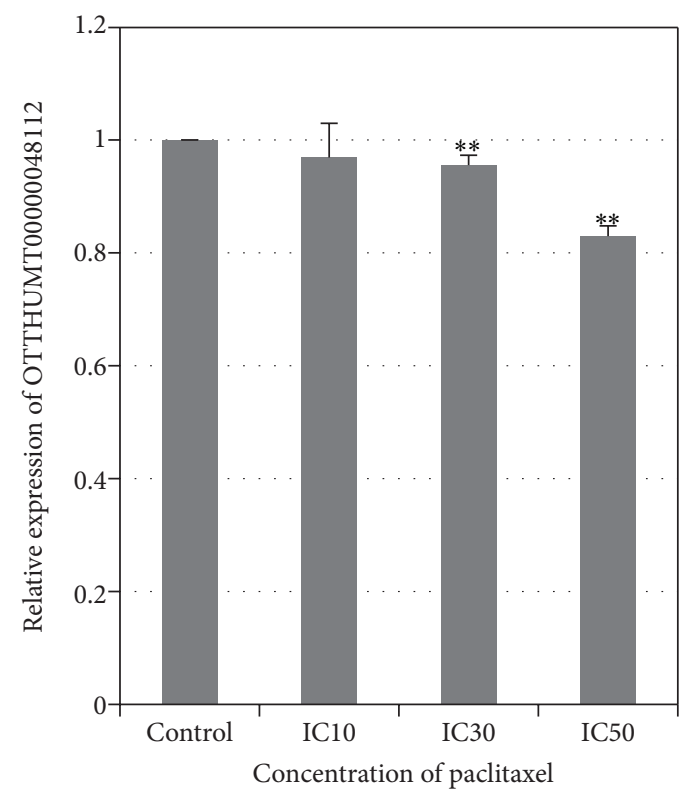

(c)

FIGURE 4: Effects of paclitaxel treatment on the expression of lncRNA in HN21B cells. (a) Dose-response curve of HN21B upon paclitaxel treatment. (b) and (c) Effect of paclitaxel treatment on the expression of OTTHUMG00000002490 and OTTHUMT00000048112, respectively. Bar, SD; $n=3 ;{ }^{*} P<0.05$ and ${ }^{* *} P<0.01$ by $t$-test.

lnc-MBL2-4:3 (OTTHUMT00000048112) was suppressed under paclitaxel treatment in a dose-dependent manner (Figure 4(c)).

3.5. Expression Levels of MBL2-4:3 (OTTHUMT00000048112) and Inc-AL355149.1-1 (OTTHUMG00000002490) in the Cisplatin-Resistant HN21B Cells. The cisplatin-resistant HN21B cells were developed by chronic treatment of HN21B cells with increasing concentration of cisplatin (Figure 5(a)).
The $\mathrm{IC}_{50}$ of cisplatin-resistant $\mathrm{HN} 21 \mathrm{~B}$ was $22.7 \mu \mathrm{M}$, which was obviously higher than that of the parental HN21B cells $(7.0 \mu \mathrm{M})$. Cisplatin-resistant $\mathrm{HN} 21 \mathrm{~B}$ cells displayed spindle-like changes in cell morphology, implicating a more aggressive phenotype (Figure 5(b)). Further, the cisplatinresistant HN21B cells exhibited enhanced expression of lnc-AL355149.1-1 (OTTHUMG00000002490) and reduced expression of lnc-MBL2-4:3 (OTTHUMT00000048112) in comparison with parental HN21B cells (Figure 5). 


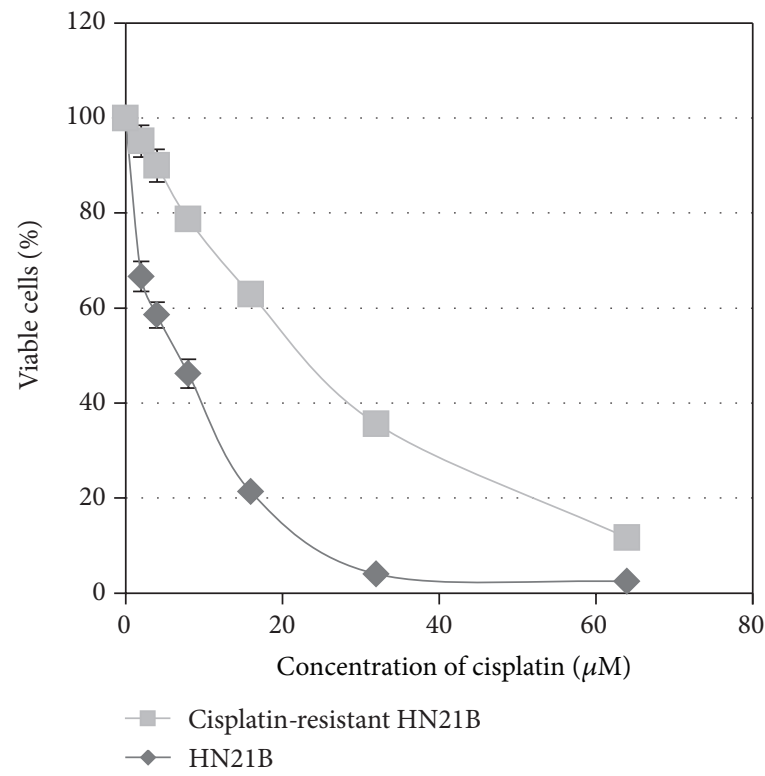

(a)

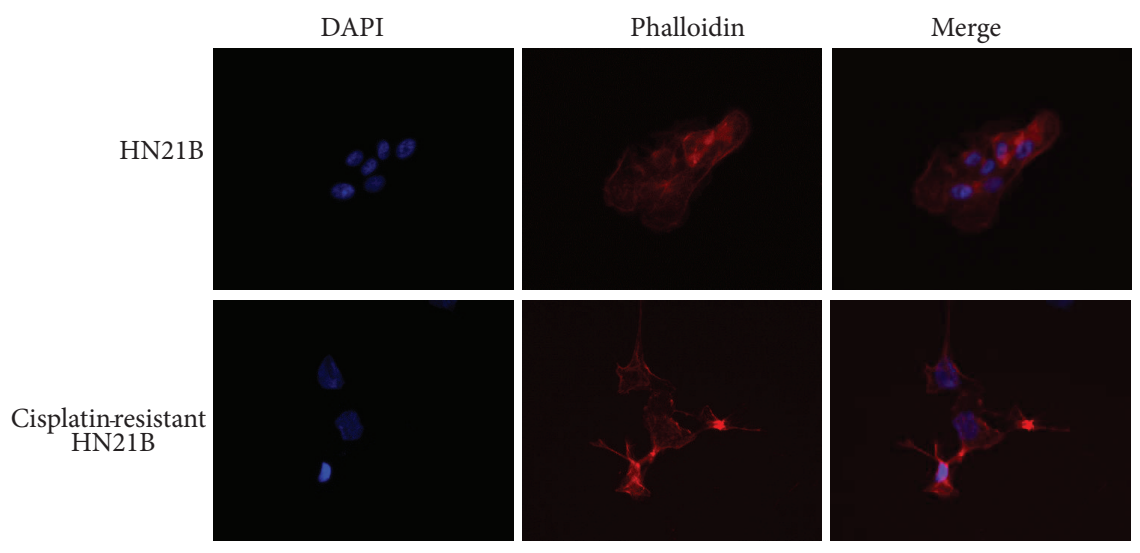

(b)

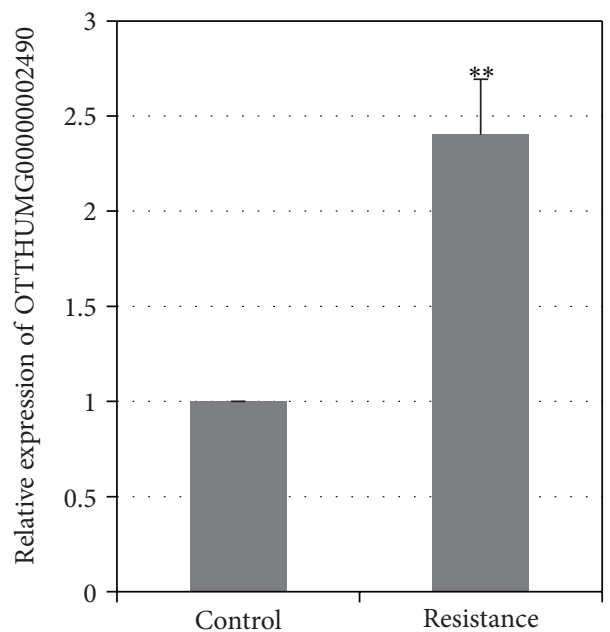

(c)

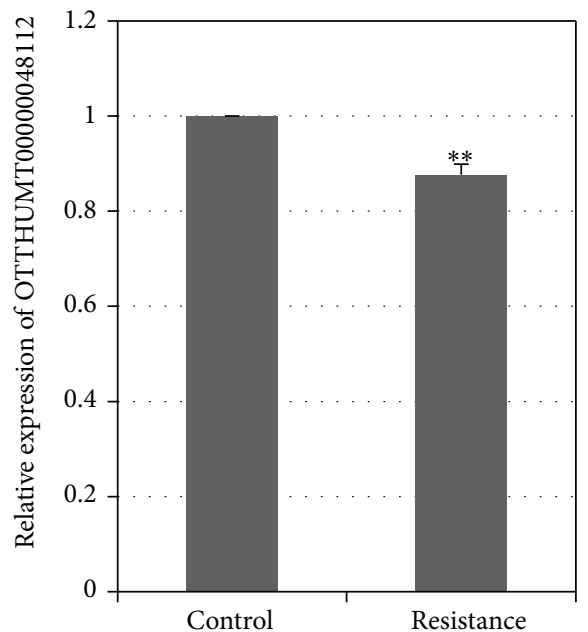

(d)

FIGURE 5: Differentially expressed lncRNA in cisplatin-resistant HN21B cells. (a) Dose-response curve of HN21B and cisplatin-resistant HN21B cells upon cisplatin treatment. (b) Morphology of HN21B and cisplatin-resistant HN21B cells. The nucleus was stained by bluefluorescent DAPI; F-actin was labeled in red with Alexa Fluor 635 phalloidin. (c) and (d) Expression of OTTHUMG00000002490 and OTTHUMT00000048112 in HN21B and cisplatin-resistant HN21B cells. Bar, SD; $n=3 ;{ }^{* *} P<0.01$ by $t$-test. 


\section{Discussion}

Expression alterations of lncRNA have been reported in several human tumors. In the past, lncRNA was regarded as functionless RNA fragment as it carries no protein coding information. With the characterization of functional noncoding RNA such as microRNA and PIWI-interacting RNAs (piRNAs) in the recent years, considerable attention has been dedicated to identify the active players during the progression and development of human malignancies. Although the deregulated lncRNA patterns have been identified in several cancers, such as prostate and liver cancers, little is known in oral tongue carcinoma. Using serial analysis of gene expression (SAGE), lncRNA expression has been demonstrated in the oral cavity and premalignant oral lesions located on tongue, gingiva, and buccal mucosa [11]. Recently, Fang et al. evaluated the expression patterns of lncRNA UCA1 (urothelial cancer-associated 1) in tongue squamous cell carcinoma and revealed that high UCA1 expression was linked to the migratory ability of the epithelial cancer cells and regional lymph node metastasis [12]. Another example is lncRNA MEG3 (maternally expressed gene 3). MEG3 is a lncRNA with the ability to regulate DNA methyltransferase $3 \mathrm{~B}$ and was found to be downregulated in the tongue SCC tissues [13]. Low MEG3 level was an independent prognostic indicator and was associated with poor survival of tongue SCC patients [13]. The differentially expressed lncRNA could also be detected in the saliva and was suggested to be a useful noninvasive biomarker for oral cancer detection [14].

In this study, we used the existing microarray data to explore the differentially expressed lncRNA patterns in the tongue SCC tissues. The probesets on the microarray chips were originally designed to detect the protein-coding genes. Usually, multiple probes are assigned to individual genes to cover the entire length of the transcript in order to ensure the accuracy of measurement. With the advance in our understanding of lncRNA and their sequence, it was recognized that particular probesets on the microarray chips match with the IncRNA sequence. By reannotation of the microarray for lncRNA, the expression information of the microarray dataset could be revealed for subsequent inspection [15]. To ascertain that the identified lncRNA is deregulated in the tongue SCC, we carried out real-time quantitative PCR to validate the expression discrepancy between the epithelial cancers and normal epithelia of the same patients. One of the limitations of this study is the sample size of paired tumor and normal tissue is not big enough. Of the 8 lncRNAs, 4/8 (50\%) were found to be deregulated in our tongue SCC cohort. To explore the potential role of the IncRNAs in the pathogenesis of tongue SCC, we correlated the expression levels with the clinicopathological parameters of tongue SCC patients. The association of lncRNA with T-stages and regional nodal status suggested that the lncRNA deregulation is potentially implicated in the rapid growth and high migratory property of the tongue SCC.

LncRNA could contribute to the cancer progression by controlling the process of proliferation and migration $[16,17]$. In view of the association with tumor stages
(OTTHUMG00000002490) and regional nodal status (OTTHUMT00000048112) of oral tongue patients, we suggested that lncRNA expression could possibly link to the progression of tongue squamous cell carcinoma. To explore the association, we treated the tongue cancer cell line with chemotherapeutic drugs which target highly proliferating cell and cell with high migratory potency $[18,19]$. Both OTTHUMG00000002490 and OTTHUMT0000004811 demonstrated significant changes and the expression changes were responsive to the treatment dosage of 5 -Fu and paclitaxel, revealing that the 2 lncRNAs can modulate the response of tongue cancer cells to chemotherapeutic agents. In addition, targeting lncRNA is suggested to be a feasible approach to overcome the resistance against chemotherapeutic drugs due to the observation that cancer cells expressing particular lncRNA are less responsive to the cytotoxic drugs [20, 21]. For example, in non-small cell lung cancer, it has been reported that cancer cells with high lncRNA AK126698 expression are associated with the cisplatin resistant phenotype [22]. As cisplatin-resistance is a challenge to the treatment efficacy of tongue squamous cell carcinoma, we developed a cisplatin-resistant model to examine the potential implication of the $2 \operatorname{lncRNAs}$ to the development of resistant phenotype in oral tongue cancers. The significant changes in OTTHUMG00000002490 and OTTHUMT0000004811 observed in the resistant cell line indicated the potential association with the development of cisplatin resistance in tongue SCC.

\section{Conclusions}

In conclusion, our results indicated that oncogenic/tumorsuppressing lncRNA could potentially be identified through computational exploration of the existing microarray data. The associations of IncRNA with the clinical features of tongue SCC patients substantiate the claim that IncRNA deregulation is linked to the biology activity of tongue SCC. Whether the deregulated lnRNA could possibly be used as diagnostic/prognostic indicators has yet to be elucidated. Further studies are warranted to decipher the functional roles of lncRNA in the pathogenesis of tongue SCC.

\section{Conflict of Interests}

The authors declare that they have no conflict of interests regarding the publication of this paper.

\section{Acknowledgments}

The study was supported by Seed Funding of Basic Research, The University of Hong Kong, and Hong Kong Area of Excellent Scheme, Hong Kong UGC.

\section{References}

[1] R. Siegel, D. Naishadham, and A. Jemal, "Cancer statistics, 2012," CA: Cancer Journal for Clinicians, vol. 62, no. 1, pp. 10-29, 2012. 
[2] J. Jalouli, M. M. Jalouli, D. Sapkota, S. O. Ibrahim, P.-A. Larsson, and L. Sand, "Human papilloma virus, herpes simplex virus and Epstein Barr virus in oral squamous cell carcinoma from eight different countries," Anticancer Research, vol. 32, no. 2, pp. 571$580,2012$.

[3] O. T. Okuyemi, J. F. Piccirillo, and E. Spitznagel, “TNM staging compared with a new clinicopathological model in predicting oral tongue squamous cell carcinoma Survival," Head \& Neck, 2014.

[4] A. Ferlito, A. Rinaldo, K. O. Devaney et al., "Prognostic significance of microscopic and macroscopic extracapsular spread from metastatic tumor in the cervical lymph nodes," Oral Oncology, vol. 38, no. 8, pp. 747-751, 2002.

[5] H. Keski-Säntti, T. Atula, J. Tikka, J. Hollmén, A. A. Mäkitie, and I. Leivo, "Predictive value of histopathologic parameters in early squamous cell carcinoma of oral tongue," Oral Oncology, vol. 43, no. 10, pp. 1007-1013, 2007.

[6] H. Bierhoff, K. Schmitz, F. Maass, J. Ye, and I. Grummt, "Noncoding transcripts in sense and antisense orientation regulate the epigenetic state of ribosomal RNA genes," Cold Spring Harbor Symposia on Quantitative Biology, vol. 75, pp. 357-364, 2010.

[7] J. Y. Tang, J. C. Lee, Y. T. Chang et al., "Long noncoding RNAs-related diseases, cancers, and drugs," The Scientific World Journal, vol. 2013, Article ID 943539, 7 pages, 2013.

[8] M. A. Chaudhry, "Small nucleolar RNA host genes and long non-coding RNA responses in directly irradiated and bystander cells," Cancer Biotherapy \& Radiopharmaceuticals, vol. 29, no. 3, pp. 135-141, 2014.

[9] Y. Yang, H. Li, S. Hou, B. Hu, J. Liu, and J. Wang, "The noncoding RNA expression profile and the effect of lncRNA AK126698 on cisplatin resistance in non-small-cell lung cancer cell," PLoS ONE, vol. 8, no. 5, Article ID e65309, 2013.

[10] Q. Liao, H. Xiao, D. Bu et al., "NcFANs: a web server for functional annotation of long non-coding RNAs," Nucleic Acids Research, vol. 39, no. 2, pp. W118-W124, 2011.

[11] E. A. Gibb, K. S. S. Enfield, G. L. Stewart et al., "Long noncoding RNAs are expressed in oral mucosa and altered in oral premalignant lesions," Oral Oncology, vol. 47, no. 11, pp. 10551061, 2011.

[12] Z. Fang, L. Wu, L. Wang, Y. Yang, Y. Meng, and H. Yang, "Increased expression of the long non-coding RNA UCA1 in tongue squamous cell carcinomas: a possible correlation with cancer metastasis," Oral Surgery, Oral Medicine, Oral Pathology, Oral Radiology, vol. 117, no. 1, pp. 89-95, 2014.

[13] L. F. Jia, S. B. Wei, Y. H. Gan et al., "Regulation and roles of MiR-26a and MEG3 in tongue squamous cell carcinoma," International Journal of Cancer, 2013.

[14] H. Tang, Z. Wu, J. Zhang, and B. Su, "Salivary lncRNA as a potential marker for oral squamous cell carcinoma diagnosis," Molecular Medicine Reports, vol. 7, no. 3, pp. 761-766, 2013.

[15] S. K. Michelhaugh, L. Lipovich, J. Blythe, H. Jia, G. Kapatos, and M. J. Bannon, "Mining Affymetrix microarray data for long non-coding RNAs: altered expression in the nucleus accumbens of heroin abusers," Journal of Neurochemistry, vol. 116, no. 3, pp. 459-466, 2011.

[16] Y. Shi, J. Lu, J. Zhou et al., "Long non-coding RNA Loc554202 regulates proliferation and migration in breast cancer cells," Biochemical and Biophysical Research Communications, vol. 446, no. 2, pp. 448-453, 2014.

[17] Y. Zhao, Q. Guo, J. Chen, J. Hu, S. Wang, and Y. Sun, "Role of long non-coding RNA HULC in cell proliferation, apoptosis and tumor metastasis of gastric cancer: a clinical and in vitro investigation," Oncology Reports, vol. 31, no. 1, pp. 358-364, 2014.

[18] P. M. de Angelis, D. H. Svendsrud, K. L. Kravik, and T. Stokke, "Cellular response to 5-fluorouracil (5-FU) in 5-FUresistant colon cancer cell lines during treatment and recovery," Molecular Cancer, vol. 5, article 20, 2006.

[19] M. Ogasawara, T. Matsubara, and H. Suzuki, "Screening of natural compounds for inhibitory activity on colon cancer cell migration," Biological and Pharmaceutical Bulletin, vol. 24, no. 6, pp. 720-723, 2001.

[20] Y. Fan, B. Shen, M. Tan et al., "Long non-coding RNA UCA1 increases chemoresistance of bladder cancer cells by regulating Wnt signaling," FEBS Journal, vol. 281, no. 7, pp. 1750-1758, 2014.

[21] Z. Liu, M. Sun, K. Lu et al., "The long noncoding RNA HOTAIR contributes to cisplatin resistance of human lung adenocarcinoma cells via downregualtion of p21(WAF1/CIP1) expression," PLoS ONE, vol. 8, no. 10, Article ID e77293, 2013.

[22] Y. Yang, H. Li, S. Hou, B. Hu, J. Liu, and J. Wang, "The noncoding RNA expression profile and the effect of lncRNA AK126698 on cisplatin resistance in non-small-cell lung cancer cell," PLoS ONE, vol. 8, no. 5, Article ID e65309, 2013. 


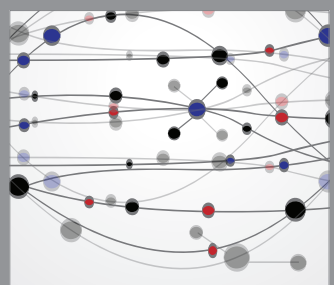

The Scientific World Journal
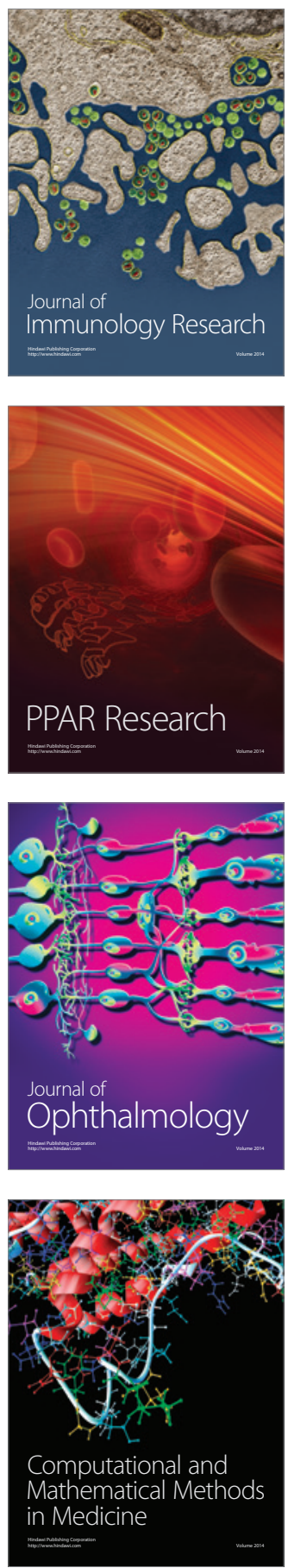

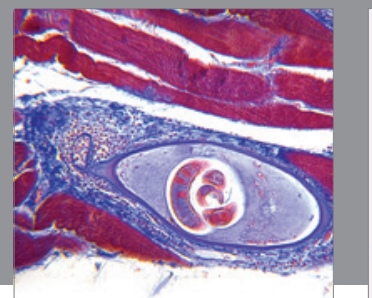

Gastroenterology

Research and Practice
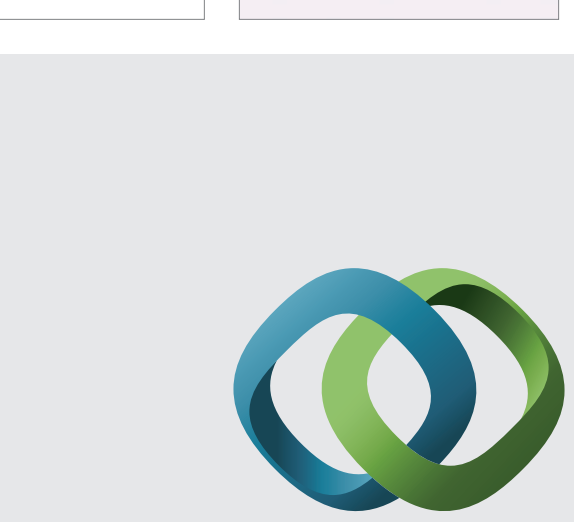

\section{Hindawi}

Submit your manuscripts at

http://www.hindawi.com
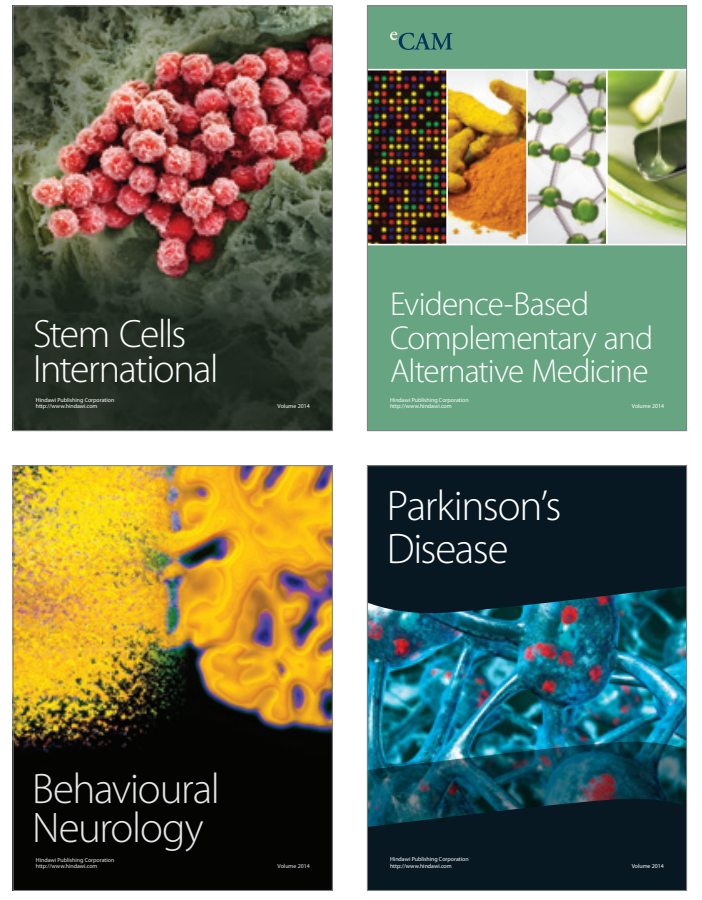
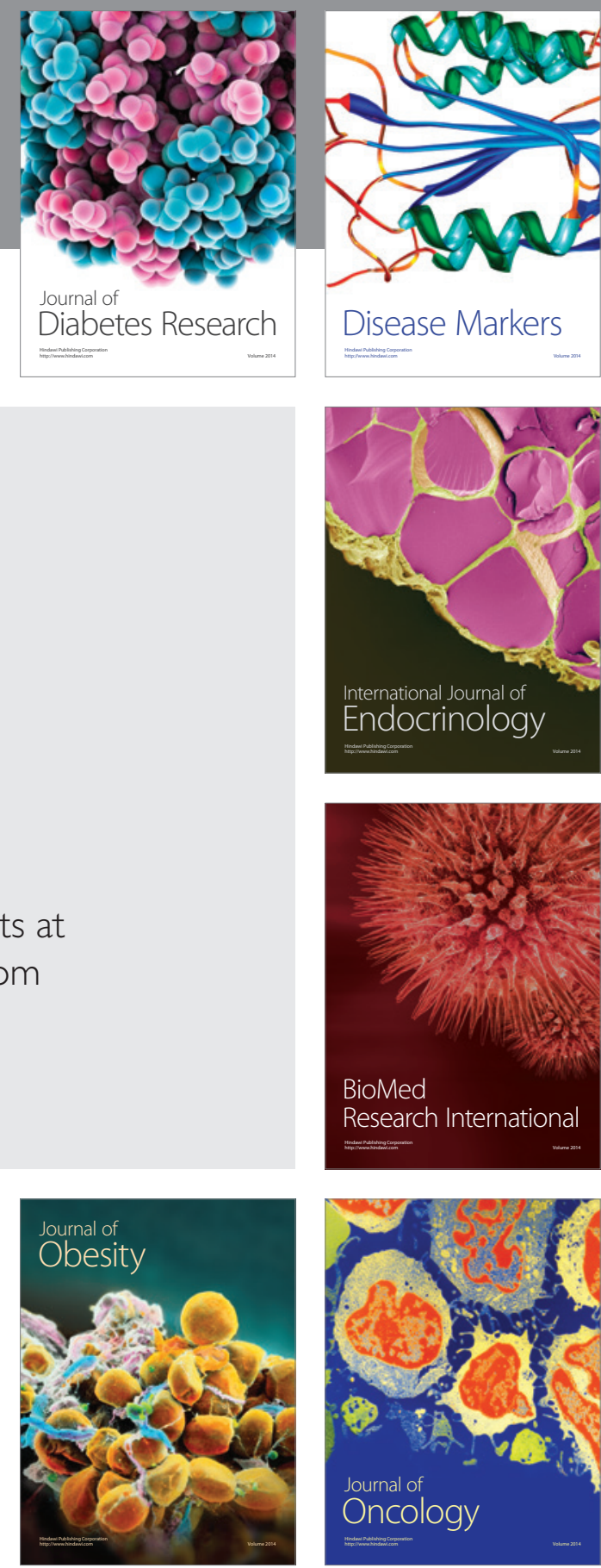

Disease Markers
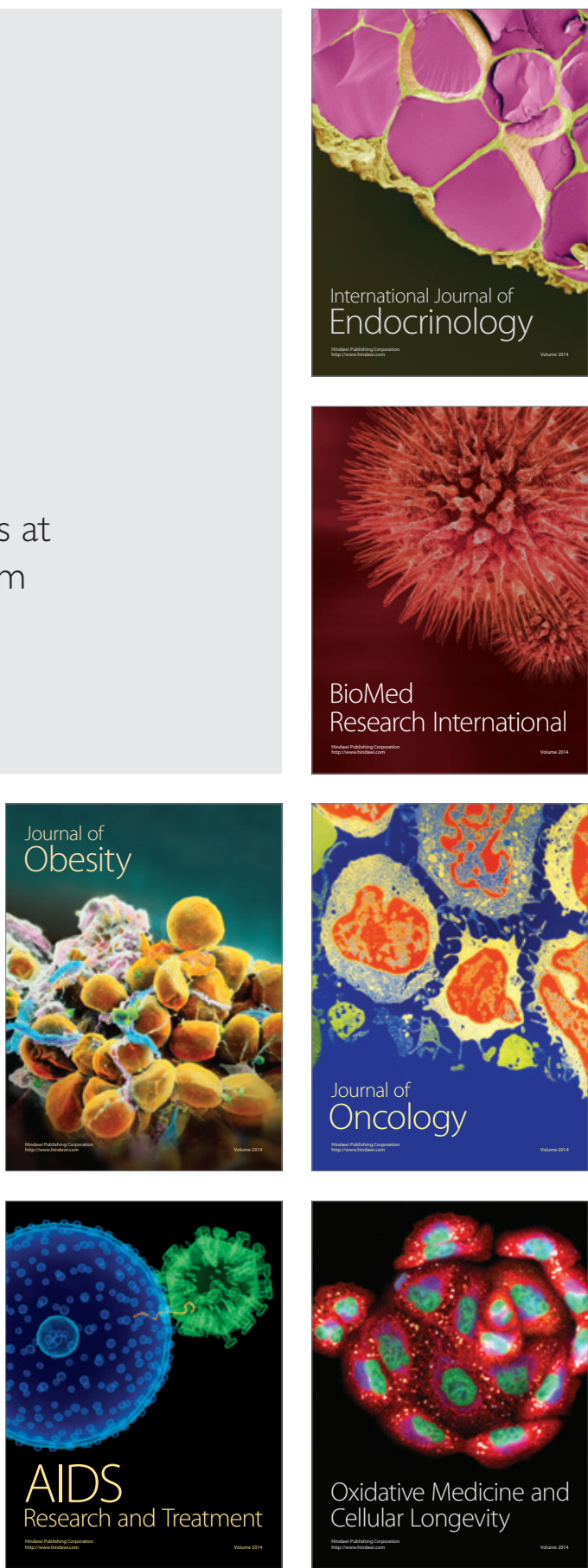